\title{
N-Acetylcysteine Normalizes Neurochemical Changes in the Glutathione-Deficient Schizophrenia Mouse Model During Development
}

\author{
Joao Miguel das Neves Duarte, Anita Kulak, Mehdi Mohammad Gholam-Razaee, Michel Cuenod, \\ Rolf Gruetter, and Kim Quang Do
}

Background: Glutathione (GSH) is the major cellular redox-regulator and antioxidant. Redox-imbalance due to genetically impaired GSH synthesis is among the risk factors for schizophrenia. Here we used a mouse model with chronic GSH deficit induced by knockout (KO) of the key GSH-synthesizing enzyme, glutamate-cysteine ligase modulatory subunit (GCLM).

Methods: With high-resolution magnetic resonance spectroscopy at $14.1 \mathrm{~T}$, we determined the neurochemical profile of GCLM-KO, heterozygous, and wild-type mice in anterior cortex throughout development in a longitudinal study design.

Results: Chronic GSH deficit was accompanied by an elevation of glutamine (Gln), glutamate (Glu), Gln/Glu, N-acetylaspartate, myoInositol, lactate, and alanine. Changes were predominantly present at prepubertal ages (postnatal days 20 and 30). Treatment with $\mathrm{N}$-acetylcysteine from gestation on normalized most neurochemical alterations to wild-type level.

Conclusions: Changes observed in GCLM-KO anterior cortex, notably the increase in Gln, Glu, and Gln/Glu, were similar to those reported in early schizophrenia, emphasizing the link between redox imbalance and the disease and validating the model. The data also highlight the prepubertal period as a sensitive time for redox-related neurochemical changes and demonstrate beneficial effects of early $\mathrm{N}$-acetylcysteine treatment. Moreover, the data demonstrate the translational value of magnetic resonance spectroscopy to study brain disease in preclinical models.

Key Words: Glutathione, MRS, N-acetylcysteine, neurochemical profile, oxidative stress, schizophrenia

$\mathrm{O}$ xidative stress and impaired redox-regulation occur in neurodegenerative diseases such as Parkinson's (1), Alzheimer's (2), and Huntington disease (3), as well as in psychiatric pathologies including bipolar disorder (4) and schizophrenia (5). Whereas redox-dysregulation in neurodegenerative diseases is likely a downstream consequence of other primary causes, compelling data suggest that redox-dysregulation is a primary risk factor for schizophrenia (5). Indeed, glutathione (GSH), the major cellular redox-regulator and antioxidant, is decreased in cerebrospinal fluid, medial prefrontal cortex $(6,7)$ and postmortem striatum (8) of schizophrenia patients. Furthermore, the two genes coding for the key GSH-synthesizing enzyme, glutamate-cysteine ligase catalytic (GCLC) and modifier (GCLM) subunits, are associated with schizophrenia $(9,10)$. Polymorphisms of trinucleotide repeats on the GCLC gene are accompanied by reduced enzyme activity, decreased GSH levels, and higher risk for the disease (10). Moreover, the response to an oxidative stress challenge in patient fibroblasts is depressed (10), indicating a deficit in GSH regulation. Thus, in combination with environmental insults that generate oxidative stress, this redox dysregulation may play a central role in schizophrenia $(5,11)$.

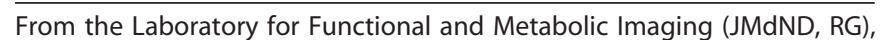
Center for Biomedical Imaging, Ecole Polytechnique Federale; Faculty of Biology and Medicine (JMdND), University of Lausanne; Center for Psychiatric Neuroscience (AK, MC, KQD), University Hospital Lausanne; and Center for Psychiatric Epidemiology and Psychopathology (MMG-R), University Hospital Lausanne; and the Department of Radiology (RG), Universities of Lausanne and Geneva, Switzerland.

Authors JMdND and AK contributed equally to this work.

Address correspondence to Kim Q. Do, Ph.D., Center for Psychiatric Neuroscience, Schizophrenia Research Unit, Site de Cery, 1008 Prilly-Lausanne, Switzerland; E-mail: kim.do@chuv.ch.

Received Mar 23, 2011; revised Jul 11, 2011; accepted Jul 29, 2011.
To investigate the link between redox imbalance and the disease, we study mice with genetic deletion (knockout) of the GCLM gene (KO) (12), which display a sustained GSH deficit compared with wild-type mice (WT) $(12,13)$. GCLM-KO mice exhibit phenotypic anomalies that are similar to those observed in patients: impaired parvalbumine (PV) immunoreactive fastspiking (FS) gamma-aminobutyric acid (GABA)ergic interneurons and oscillatory network activity in the ventral hippocampus (13) and anterior cingulate cortex (ACC) (14), deficits in myelination (15), and alterations in social and stress-related behaviors (14). These deficits are aggravated by additional oxidative stress during particular developmental periods (14). This is in line with the notion that schizophrenia is a neurodevelopmental disease involving progressive deterioration during childhood and adolescence $(16,17)$.

Schizophrenia patients display brain neurochemical alterations, as revealed by in vivo magnetic resonance spectroscopy (MRS). A meta-analysis revealed consistently decreased $\mathrm{N}$-acetylaspartate (NAA) concentrations across different brain regions (18). This finding has received further support from recent studies in hippocampus (19), striatum $(20,21)$, thalamus (22), and ACC (23). Others have demonstrated increased NAA levels in hippocampus of chronic patients (24) and prefrontal cortex of high-risk adolescents (21). Alterations are also frequently reported for glutamine (Gln), glutamate (Glu), and GIn/Glu. Like the case for NAA, the direction of the changes is variable. Accordingly, increased levels of Gln, Glu, or Gln/Glu have been found in early stages of the disease (23,25-27), whereas decreased levels have been observed in chronic patients $(28,29)$. MRS can be applied both in clinical subjects and preclinical animal models. As such, it represents a methodology of choice for translational approaches. Indeed, a recent preclinical MRS study reported elevated GIn/Glu and altered brain glucose and lactate metabolism in prefrontal cortex of rats with $\mathrm{N}$-methyl-D-aspartate receptor (NMDAR) hypofunction (30), a model that mimics schizophrenia symptoms $(31,32)$. 
Here we used state-of-the-art high-resolution MRS at 14.1 T in a preclinical setting to investigate noninvasively whether redox imbalance induces neurochemical alterations in GCLM-KO anterior cortex similar to those observed in patients. In contrast to most clinical studies, which routinely quantify concentrations of only few metabolites at one time point of the disease, we measured a profile of 18 metabolites in a longitudinal study design (33). Thus, one objective was to screen the GCLM-KO mouse brain at various stages of development for relevant neurochemical changes to pinpoint sensitive periods. Because GSH depletion in GCLM-KO mice was associated with schizophrenia-relevant neurochemical alterations, another objective was to test whether partially restoring the redox balance in these animals with $\mathrm{N}$-acetylcysteine (NAC) (34) would normalize their neurochemical profile. NAC serves as precursor for GSH synthesis (35) and exerts direct antioxidant effects (36). Given to patients as adjunct to their antipsychotic therapy, NAC alleviates negative symptoms, reduces side effects (37), and improves a preattentional component of the auditory evoked potential on electroencephalogram (38).

\section{Methods and Materials}

\section{Animals}

GCLM-KO animals were generated (12) and kindly provided by TP Dalton (Cincinnati University, Ohio). Mice used for this study were bred and maintained in the local animal facility in a temperature- and humidity-controlled environment under a 12-h lightdark cycle with free access to food and water. All experiments were performed in accordance with the Guide for the Care and Use of Laboratory Animals (39) and were approved by the cantonal veterinary office.

\section{Design}

The first experiment investigated whether the developing anterior cortical neurochemical profile differed between GCLM genotypes. A second experiment assessed whether NAC treatment normalizes the observed genotype differences. To account for testretest reliability, a non-NAC-treated control group for each genotype was included in the second experiment. This resulted in overall more non-NAC-treated versus NAC-treated animals. Respective animal numbers are as follows: 27 GCLM-KO (17 nontreated; 10 NACtreated), 25 heterozygous ( $\mathrm{HZ} ; 19$ nontreated; 6 NAC-treated), and 32 WT ( 20 nontreated; 12 NAC-treated) mice. In both experiments, a mixed cohort of male and female mice was used (the number of males and females was balanced in each experimental group).

\section{${ }^{1}$ H-Magnetic Resonance Spectroscopy}

In vivo ${ }^{1} \mathrm{H}-\mathrm{MRS}$ has been implemented on clinical platforms to allow the quantification of neurochemical profiles comprising 14 metabolites $(40,41)$. It has also been validated for rodents to determine a profile of 20 metabolites (42). Here, we quantified 18 metabolites at postnatal Days (P)10, P20, P30, P60, and P90, using a DirectDrive spectrometer (Varian, Palo Alto, California) interfaced to an actively shielded 14.1-T magnet with a 26-cm horizontal bore (Magnex Scientific, Abingdon, United Kingdom) and a quadrature ${ }^{1} \mathrm{H}$ coil, as previously described (33). Briefly, field homogeneity was achieved with FAST(EST)MAP $(43,44)$ and spectra were acquired using SPECIAL (45) with echo time of $2.8 \mathrm{msec}$, repetition time of 4 $\mathrm{sec}$ from the anterior cortex (voxel of interest at .62 relative to bregma; volume ranged from 2.5 to $3.8 \mu \mathrm{L}$ across ages) of mice under isoflurane (Attane, Minrad, New York) anesthesia. Metabolite concentrations were quantified with LCModel (Stephen Provencher, Oakville, Canada) including a macromolecule spectrum in the database and scaling concentrations to corrected water content as measured from unsuppressed water spectra (33). Effects of repeated isoflurane anesthesia on the neurochemical profile and on behavior were investigated previously and can be largely ruled out (33).

\section{NAC Treatment}

NAC (Fluimucil, Zambon, Switzerland) was administered in drinking water at $2.4 \mathrm{~g} / \mathrm{L}(480 \mathrm{mg} / \mathrm{kg} \mathrm{NAC}$ per adult animal; calculated based on an average water consumption of $5 \mathrm{~mL} /$ animal/day and average animal body weight of $25 \mathrm{~g}$; comparable to Atkuri et al [34]) before and during mouse pregnancy and after birth throughout the experimental period. Fresh solutions were prepared every other day. Average daily drinking volume per adult animal was significantly lower in NAC-treated animals across all three genotypes $(4.78 \mathrm{~mL} \pm$ SEM .19) compared with non-NAC-treated control animals (6.69 $\mathrm{mL} \pm$ SEM .27; $p<.001 ; n=30$ ) but was within the normal range compared with other studies (46).

\section{Blood Glutathione Measurements}

To assess whether NAC consumed by the dam is transmitted to her suckling pups, blood samples were collected from offspring of NAC-treated and non-NAC-treated dams at P10. Samples were also collected at P30, by which age animals drink NAC in water on their own. Increased blood GSH levels were taken as indirect measure of a NAC effect. Accordingly, GCLM-KO (P10 nontreated: $n=6$; NACtreated: $n=5$; P30 nontreated: $n=8$; NAC-treated: $n=7)$ and WT (P10 nontreated: $n=6$; NAC-treated: $n=4$; P30 nontreated: $n=$ 7; NAC-treated: $n=8$ ) animals were decapitated. Trunk blood was collected in ethylenediamine tetraacetate K3E microvettes (Sarstedt, Nuembrecht, Germany), shock frozen on dry ice, and stored at $-80^{\circ} \mathrm{C}$. Total GSH concentration in whole blood was determined using a colorimetric assay from Calbiochem (EMD Biosciences, Darmstadt, Germany), with a sensitivity of $5 \mu \mathrm{mol} / \mathrm{mL}$. Samples were analyzed in triplicates (intraassay variation coefficient of $3.42 \%$; interassay variation coefficient of $3.58 \%$ ).

\section{Statistical Analysis}

A multiple regression was fitted for each metabolite at each time point to compare metabolite concentrations between the three genotypes using R environment (The R project for Statistical Computing); Bonferroni adjustment (for five time points) was applied to each comparison to avoid the inflation of Type I error. Animals' sex was controlled for in all regression models. Fit adequacy was assessed for each multiple regression (47). To test whether NAC treatment had normalizing effects, metabolite concentrations of NACtreated $\mathrm{KO}$ and $\mathrm{HZ}$ animals were compared with non-NAC-treated WT animals at relevant time points (i.e., when metabolites had been significantly different from WT in non-NAC-treated condition) with two-way analysis of variance (ANOVA) considering sex as the second factor. The effect of NAC within genotypes was assessed for each metabolite and time point using two-way ANOVA (treatment $\times$ gender) with Bonferroni adjustment for the five time points. To assess whether blood GSH levels differed between genotypes, one-way ANOVA was performed for NAC- and non-NACtreated animals at P10 and P30 including samples of both genders. Significant genotype effects were followed up with independentsample $t$ tests. Whether blood GSH levels were increased in NACversus non-NAC-treated $\mathrm{WT}$ and $\mathrm{HZ}$ animals at P10 was assessed with independent-sample $t$ tests. Average drinking volume of nonNAC-treated versus NAC-treated animals was analyzed with an independent-sample $t$ test including data of all genotypes and both genders. The ANOVA and $t$ tests were performed in PASW Statistics 18 (SPSS, Somers, New York). 


\section{Results}

Spectra allowed quantifying a profile of 18 metabolites with Cramer-Rao lower bound (CRLB) below 25\% with few exceptions for metabolites that occur at low concentration in cortex. The CRLB was above $25 \%$ for four measurements of $\mathrm{N}$-acetylaspartylglutamate between P20 and P60 (at maximum 48\%) and of aspartate (Asp) at P10 (at maximum 31\%). GSH concentrations in the cortex of GCLM-KO mice were very low and below the detection limit and thus associated with high CRLB at all time points. As observed in Figure 1, the neurochemical profile of all genotypes generally changed as function of development (33).

\section{Neurochemical Alterations in GCLM-KO and HZ Mice}

GCLM-KO Mice. The analyses revealed age-specific neurochemical alterations in GCLM-KO cortex relative to WT (Figure 1A "nontreated"; summarized in Table 1). As expected, spectra of KO mice showed substantially decreased GSH levels across all ages (at least $-83 \%$ relative to $\mathrm{WT}$ [at $\mathrm{P} 10 ; \beta=-.7, t(47)=-7.6, p<0.005$ ]. This GSH deficit induced prominent neurochemical alterations at prepubertal age P20 as exemplified in Figure 2A: an increase of
$\mathrm{G} \ln [+35 \% ; \beta=1.01, t(49)=4.09, p<.005], \mathrm{Glu}[+12 \% ; \beta=1.05$, $t(49)=2.95, p<.05]$, and Gln/Glu $[+23 \% ; \beta=.98, t(49)=3.08, p<$ .05], as well as of myo-inositol [Ins; $+24 \% ; \beta=.5, t(49)=2.89, p<$ $.05]$ and NAA [ $+14 \% ; \beta=.99, t(49)=3.38, p<.05]$ relative to WT. $\mathrm{G} \ln [+34 \% ; \beta=.95, t(49)=2.74, p<.05]$ and $\mathrm{Gln} / \mathrm{Glu}[+21 \% ; \beta=$ $.65, t(49)=3.16, p<.05]$ remained elevated at P30. At adult age $\mathrm{P} 90$, the concentrations of lactate $[\mathrm{Lac} ;+68 \% ; \beta=1.15, t(48)=$ $4.04, p<.005]$ and alanine [Ala; $+28 \% ; \beta=.3, t(48)=2.9, p<.05]$ were increased. As shown in Figure 1B ("nontreated"), the other metabolites remained unaffected by GSH deficit.

GCLM-HZ Mice. Compared with WT, the spectra of $\mathrm{HZ}$ animals displayed a slight reduction of cortical GSH concentration at most developmental ages with a significant decrease at P60 [-24\%; $\beta=$ $-.28, t(48)=-3.58, p<.05]$. This slight but consistent GSH deficit was accompanied by an elevation of $G \ln [+23 \% ; \beta=.74, t(49)=$ $3.01, p<.05]$ and a decrease in Ala $[+24 \% ; \beta=-.34, t(49)=-2.73$, $p<.05$ ] at P20. See Figure 1A ("nontreated"; summarized in Table 1).

\section{NAC Treatment}

NAC Affects the Neurochemical Profile of All Genotypes. Within-genotype comparisons revealed NAC-induced neurochem-

WT

HZ KO
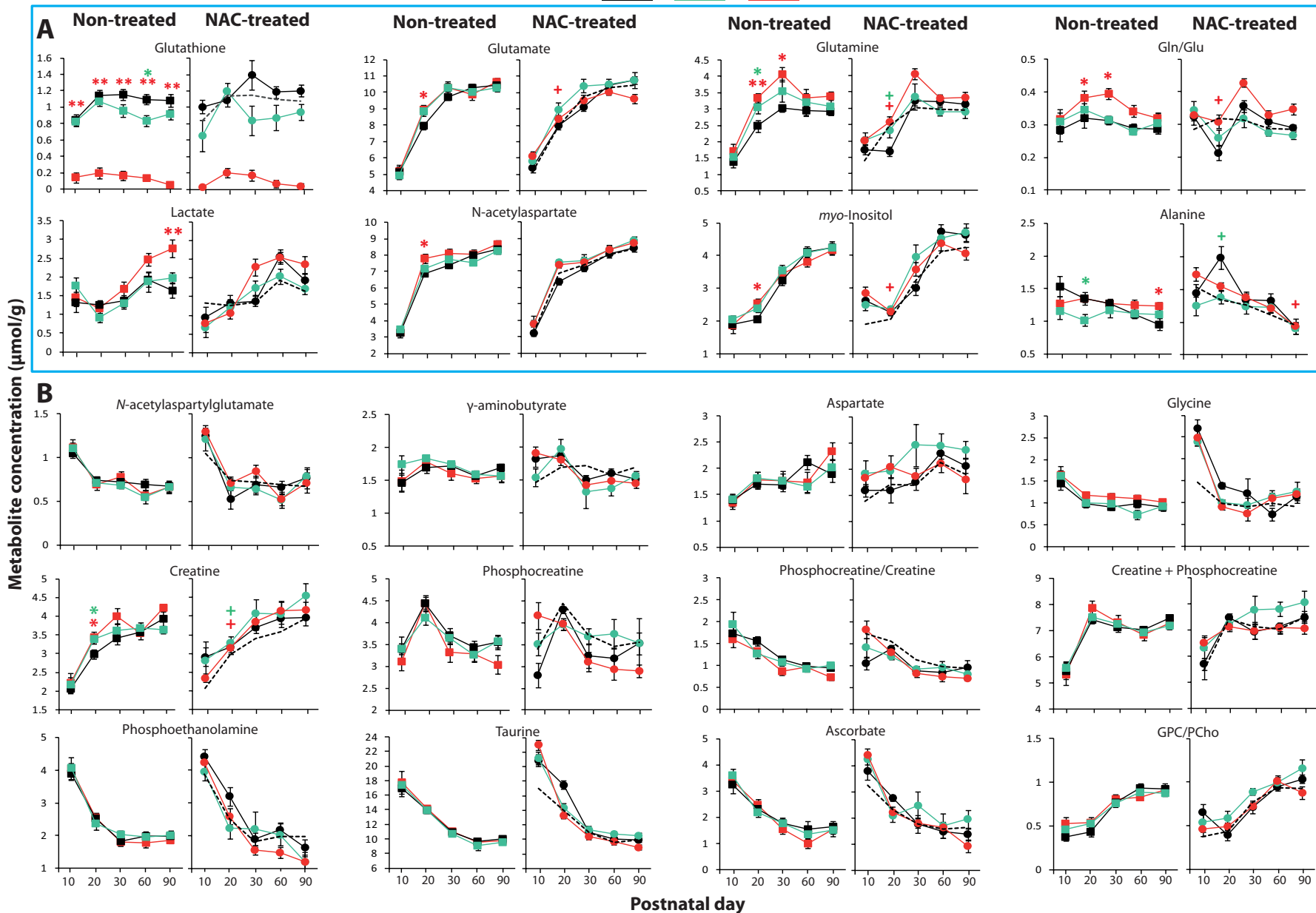

Figure 1. Cortical neurochemical profile of nontreated control and N-acetylcysteine (NAC)-treated GCLM wild type (WT), heterozygous (HZ), and knockout (KO) animals throughout development. Data are represented as mean \pm SEM for each group. The dotted line representing mean concentration levels of nontreated WT animals was included to facilitate visual evaluation of NAC treatment effects. $\left({ }^{*}{ }^{* *}\right)$ indicates significant concentration differences between GCLM-KO and WT (red) and GCLM-HZ and WT (green). ${ }^{*} .005<p<.05 .{ }^{* *} p<.005 .{ }^{+}$Indicates that NAC treatment normalized the metabolite concentration to nontreated WT level for KO (red) and $\mathrm{HZ}$ (green) animals. 
Table 1. Neurochemical Alterations in Anterior Cortex of GCLM-KO and HZ Mice Relative to WT and the Effect of NAC

\begin{tabular}{|c|c|c|c|c|c|c|c|c|c|c|}
\hline & \multicolumn{2}{|c|}{ P10 } & \multicolumn{2}{|c|}{$P 20$} & \multicolumn{2}{|c|}{ P30 } & \multicolumn{2}{|c|}{$P 60$} & \multicolumn{2}{|c|}{ P90 } \\
\hline & $\mathrm{HZ}$ & $\mathrm{KO}$ & $\mathrm{HZ}$ & $\mathrm{KO}$ & $\mathrm{HZ}$ & $\mathrm{KO}$ & $\mathrm{HZ}$ & $\mathrm{KO}$ & $\mathrm{HZ}$ & KO \\
\hline Glutathione & & $\downarrow \downarrow 83 \%$ & & $\downarrow \downarrow 83 \%$ & & $\downarrow \downarrow 85 \%$ & $\downarrow 24 \%$ & $\downarrow \downarrow 88 \%$ & & $\downarrow \downarrow 95 \%$ \\
\hline Glutamate & & & & $\uparrow 12 \%$ & & & & & & \\
\hline Glutamine & & & $\uparrow 23 \%$ & $\uparrow \longdiv { \uparrow 3 5 \% }$ & & $\uparrow 34 \%$ & & & & \\
\hline Gln/Glu & & & & $\uparrow 23 \%$ & & $\uparrow 21 \%$ & & & & \\
\hline N-Acetylaspartate & & & & $\uparrow 14 \%$ & & & & & & \\
\hline Lactate & & & & & & & & & & $\uparrow \uparrow 68 \%$ \\
\hline myo-Inositol & & & & $\uparrow 24 \%$ & & & & & & \\
\hline Alanine & & & $\downarrow 24 \%$ & & & & & & & $\uparrow 28 \%$ \\
\hline
\end{tabular}

This table summarizes significant differences between genotypes in the neurochemical profile of KO and HZ animals compared with WT. Moreover, it shows which of these differences between genotypes were normalized to non-NAC-treated WT level in response to chronic NAC treatment. Arrows indicate the direction of the neurochemical change (i.e., arrows pointing down indicate that this metabolite concentration is decreased relative to WT and vice versa). One arrow represents $.005<p<.05$. Two arrows reflect $p<.005$. Underlined effects were normalized by NAC treatment to nontreated WT levels.

Gln, glutamine; Glu, glutamate; $\mathrm{HZ}$, heterozygous; KO, knockout; NAC, $N$-acetylcysteine; P, postnatal day; WT, wild type.

ical changes for all genotypes (Figure 1; summarized in Table 2). These changes were most prominent in WT, which showed increased Ins $(+41 \%)$ and glycerophosphocholine/phosphocholine (GPC/PCho; $+65 \%$ ) at P10, as well as increased ascorbate (Asc; $21 \%$ ), Ala $(+24 \%)$, and taurine (Tau; $+28 \%)$ and decreased Gln $(-32 \%)$ and $\mathrm{Gln} / \mathrm{Glu}(-47 \%)$ at P20. NAC-treated HZ mice only displayed an increase in GPC/PCho ( $+33 \%)$ at P90 compared to non-NACtreated HZ. In KO mice, NAC treatment decreased GIn (-24\%) concentrations at P20 and phosphoethanolamine (PE; $-47 \%)$ at P90 in comparison to non-NAC-treated KO. See Table 2 for significance levels.

NAC Has Normalizing Effects in GCLM-KO and HZ Animals. Considering the previously identified neurochemical anomalies in GCLM-KO and $\mathrm{HZ}$ animals relative to WT, we assessed whether NAC has normalizing effects. As indicated in Figure 1A ("NAC-treated") and Table 1, NAC treatment from gestation onward normalized the concentrations of Gln, Glu, Gln/Glu, and Ins in young P20 KO animals, as well as the concentration of Ala in adult P90 KO animals to non-NAC-treated WT level. In HZ animals, NAC treatment similarly normalized GIn and Ala levels at P20. GSH concentration at P60 was not statistically more different between NAC-treated $\mathrm{HZ}$ animals and WT. Rather than being a NAC-induced normalization, we suggest this was due to relatively high intersubject variability in the NAC-treated group.

NAC Treatment of the Dam Increases Blood GSH in Preweanling WT and $\mathbf{H Z}$ Offspring. Table 3 summarizes data obtained from whole-blood GSH measurements at P10 and P30. As in the brain, GSH levels in the blood of KO (NAC- and nontreated) animals were drastically decreased compared with WT and HZ at both P10 and P30. GSH concentrations in nontreated $\mathrm{HZ}$ animals were similar to WT at both ages. Consistent with the developmental trend of cortical GSH concentrations, blood GSH levels in nontreated animals strongly increased from P10 to P30. NAC treatment significantly upregulated GSH levels in WT at P10 but not at P30. A similar trend was present in $\mathrm{HZ}$ animals. Thus, NAC increased blood GSH levels early on when endogenous GSH concentrations were still relatively low. NAC treatment had no effect on blood GSH levels in $\mathrm{KO}$ animals.

\section{Discussion}

We used ${ }^{1} \mathrm{H}-\mathrm{MRS}$ at $14.1 \mathrm{~T}$ to determine the cortical neurochemical profile of GCLM-KO, HZ, and WT mice throughout development and to investigate the effect of redox imbalance due to GSH deficit, which has been implicated in schizophrenia $(5,11)$. The high resolu- tion and sensitivity of the method allowed us to identify a profile of redox-sensitive metabolites-namely, GIn, Glu, GIn/Glu, NAA, Ins, Lac, and Ala. The majority of these metabolites were altered in young $\mathrm{KO}$ animals during the prepubertal period (P20 and P30). Exceptions were Lac and Ala, which accumulated in the adult $\mathrm{KO}$ cortex as a consequence of chronic GSH deficit. Long-term treatment with NAC normalized most neurochemical alterations to WT level.

\section{Chronic GSH Deficit Induces Neurochemical Alterations in Anterior Cortex}

Compared with WT, GCLM-KO mice displayed a severe and sustained GSH depletion in anterior cortex, which was accompanied by increased concentration of Glu, a neurochemical precursor of GSH. Notably, Glu accumulation is also observed in neuronal cultures treated with the GSH synthesis inhibitor L-buthionine-(S,R)-sulfoximine (BSO) (48). GCL enzyme function is impaired in both GCLM-KO and BSO-treated conditions. As a result, less Glu is incorporated into GSH and may accumulate, potentially leading to excitotoxicity (49) and impaired cell energy metabolism (50). Increased Glu levels are consistent with the NMDAR hypofunction theory of schizophrenia (51). NMDARs are crucial to control the normal firing rate of FS interneurons (52). NMDAR hypo-function renders FS interneurons less excitable, resulting in persistent disinhibition of pyramidal neurons and increased extracellular Glu. Interestingly, PVFS interneurons are functionally impaired in ACC of GCLM-KO mice $(5,53)$. Whether this PV interneuron impairment is associated with NMDAR hypofunction remains unclear. High Glu levels in the GCLM-KO anterior cortex are also in line with the recently reported antipsychotic efficacy of agonists for the mGluR-2 receptors, which inhibit neuronal Glu release at high extracellular Glu concentration $(54,55)$.

The GCLM-KO anterior cortex was furthermore characterized by elevated GIn and GIn/Glu. Together with increased Glu, these neurochemical alterations may reflect differences in number or metabolism of specific cell types within this region compared with WT. Thus, future studies will need to clarify whether PV deficit in GCLM-KO ACC $(5,53)$ is associated with a loss of PV FS interneurons. Elevated GIn/Glu could also be a consequence of increased extracellular synaptic Glu concentration, which stimulates the Glu-GIn cycle. Accordingly, it has been reported that GIn synthesis is potentiated in response to high extracellular Glu via a functional coupling between the glutamate transporter EAAT2 and glutamine synthase (56).

Increased concentrations of Gln, Glu, and Gln/Glu are consistent with clinical MRS data from early stages of schizophrenia. Accord- 

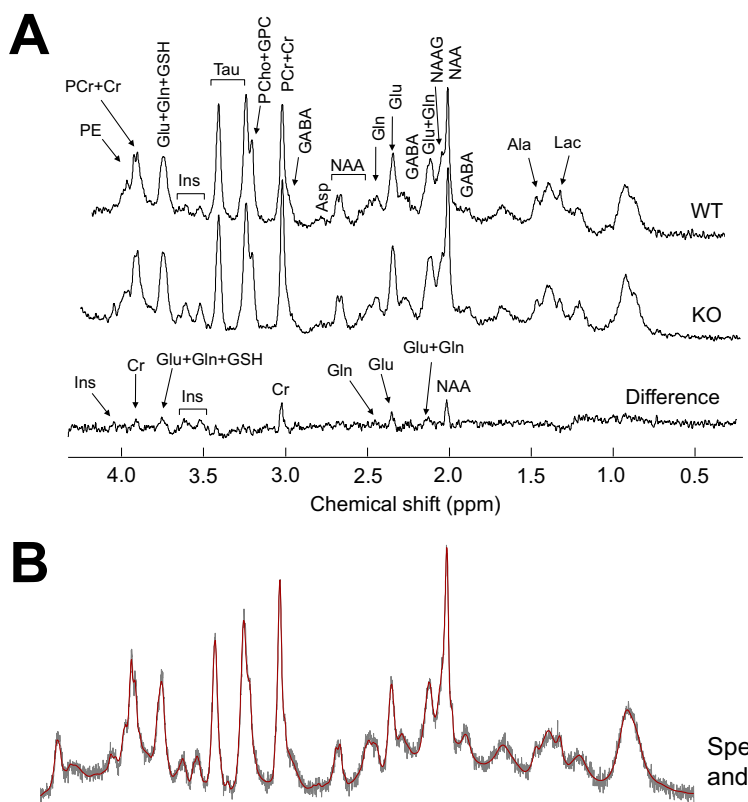

Spectrum and fitted line

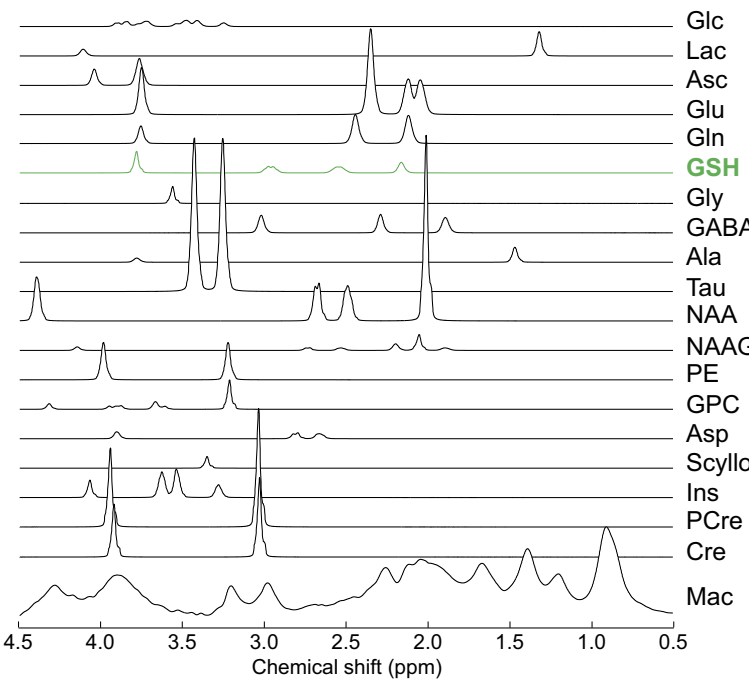

Figure 2. (A) Representative in vivo ${ }^{1} \mathrm{H}-\mathrm{MRS}$ spectra acquired at $14.1 \mathrm{~T}$ from the anterior cortex of wild type (WT) and knockout (KO) mice at postnatal day (P) 20. Most prominent alterations induced by glutathione (GSH) depletion were observed at this age and are evidenced by the difference spectrum-namely, increased NAA, Glu, Gln, Ins, and Cre concentrations. The most intense resonance of GSH at 3.77 ppm overlaps with Glu and Gln, masking the decrease of the GSH peak. For resolution enhancement, a shifted Gaussian function ( $\mathrm{gf}=.12$ and $\mathrm{gsf}=.05$ ) was applied before Fourier transformation. (B) Fit of the ${ }^{1} \mathrm{H}-\mathrm{MRS}$ spectrum from the anterior cortex of a P20 mouse anterior cortex. The LCModel fit line is shown in red over the raw spectrum. A decomposition of the fit line in spectra of the individual metabolites is shown below, notably also for GSH. Ala, alanine; Asc, ascorbate; Asp, aspartate; $\mathrm{Cr}$, creatine; GABA, $\gamma$-aminobutyrate; Gln, glutamine; Glu, glutamate; GSH, glutathione; Gly, glycine; GPC, glycerophosphocholine; Lac, lactate; Ins, myo-inositol; Mac, macromolecule baseline; NAA, $N$-acetylaspartate; NAAG, N-acetylaspartylglutamate; PE, phosphoethanolamine; PCho, phosphocholine; PCr, phosphocreatine; Tau, taurine.

ingly, a study with nonpsychotic adolescents at high genetic risk (25) and other studies with first-episode drug-naive (26) or minimally treated patients (23) reported increased Gln, Glu, or Gln/Glu. In contrast, studies with chronic patients frequently report a decrease in the concentrations of these metabolites $(28,29)$. This suggests that chronic illness and medication lead to a gradual progres- sion of neurochemical changes and that increased Gln, Glu, and Gln/Glu may serve as metabolic markers for the early disease state.

The anterior cortex of GCLM-KO mice also displayed increased NAA concentration. Changes in NAA concentration are observed in brains of schizophrenia patients across various regions. However, in contrast to our results, patient studies generally point toward decreased concentrations (18). It should be noted, however, that this decrease in NAA concentration is subtle and requires large sample sizes to be detected, which may be related to considerable heterogeneity among schizophrenia patients. Indeed, few studies have reported an elevation of NAA levels in the hippocampus of schizophrenia patients (23) and in prefrontal cortex of adolescents at high genetic risk (21). Our results are thus in line with a general NAA pathology in patients. In GCLM-KO mice, increased NAA levels are present at prepubertal age, which has so far not been studied in clinical settings. The prepubertal period around P20 is characterized by a high rate of myelination $(57,58)$. NAA accumulation in GCLM-KO mice may, therefore, reflect reduced deacetylation by oligodendrocytes, which is essential for the synthesis of myelin lipids (59). Impaired cortical myelination is in agreement with reduced immunoreactivity of myelin basic protein in GCLM-KO ACC compared with WT (15). Interestingly, gene expression profiling, neurocytochemical, and neuroimaging studies also strongly suggest compromised myelination in schizophrenia $(60,61)$. The increase in Ins levels at the same age, P20, may reflect a reduction in synthesis of phospholipids, its incorporation into lipid membranes, or both $(62,63)$. Altered Ins concentration has been observed in the brain of schizophrenia patients $(28,64)$ and has been linked to cognitive impairment in several neurological disorders (65-67).

At P90, chronic GSH deficit in $\mathrm{KO}$ animals resulted in a drastic accumulation of cortical Lac and increased Ala concentration, which may be related to altered glucose metabolism and mitochondrial dysfunction. Indeed, it has been demonstrated that oxidative stress diminishes mitochondrial oxidative phosphorylation and activity of the citric acid cycle enzyme alpha-ketoglutarate dehydrogenase (68), which can contribute to both Lac accumulation and increased Glu. The fact that Lac and Ala accumulate in adult $\mathrm{KO}$ but not in $\mathrm{HZ}$ animals, which experience only a transient GSH deficit, suggests that accumulation of both metabolites marks the long-term consequences of chronic GSH deficiency. Clinical studies reveal discrepant Lac and Ala changes in patients' postmortem brain tissue $(69-71)$, cerebrospinal fluid $(72,73)$ and plasma (74). Because these two metabolites have not been quantified in patients with in vivo MRS, it remains unclear how our results relate to patient cortical neurochemical data. We propose that the concentrations of Lac and Ala in the patient brain may serve as important indicators for long-term redox-related changes in future in vivo MRS studies.

\section{Neurochemical Alterations in GCLM-KO Mice Occur Primarily at Prepubertal Ages}

Prominent neurochemical alterations in GCLM-KO mice at prepubertal ages P20 and P30 suggest high GSH demand during this period. At this age, cerebral metabolism must meet energy requirements to maintain the high rate of cellular differentiation, myelination, synapse formation, and axon- and dendrite-sprouting (75). Generation of reactive oxygen species increases concomitantly. The relative lack of GSH to neutralize these reactive oxygen species leads to altered metabolism and cell injury-namely, in mitochondria. Interestingly, metabolites such as Gln, Glu, Gln/Glu, Ins, and NAA with concentrations that were changed during the prepubertal period were expressed at normal levels in adulthood. This may be related to a smaller GSH demand at adult ages. In addition, KO animals may develop adaptational or compensatory mechanisms 
Table 2. Within Genotype Effects of NAC Treatment on Metabolite Concentrations

\begin{tabular}{|c|c|c|c|c|c|c|c|c|c|c|c|c|c|c|c|}
\hline & \multicolumn{3}{|c|}{ P10 } & \multicolumn{3}{|c|}{ P20 } & \multicolumn{3}{|c|}{ P30 } & \multicolumn{3}{|c|}{ P60 } & \multicolumn{3}{|c|}{ P90 } \\
\hline & WT & $\mathrm{HZ}$ & $\mathrm{KO}$ & WT & $\mathrm{HZ}$ & $\mathrm{KO}$ & WT & $\mathrm{HZ}$ & $\mathrm{KO}$ & WT & $\mathrm{HZ}$ & $\mathrm{KO}$ & WT & $\mathrm{HZ}$ & $\mathrm{KO}$ \\
\hline Ascorbate & & & & $\uparrow 21 \%$ & & & & & & & & & & & \\
\hline Glutamine & & & & $\downarrow 32 \%$ & & $\downarrow 24 \%$ & & & & & & & & & \\
\hline Gln/Glu & & & & $\downarrow 47 \%$ & & & & & & & & & & & \\
\hline Phosphoethanolamine & & & & & & & & & & & & & & & $\downarrow 47 \%$ \\
\hline GPC/PCho & $\uparrow 65 \%$ & & & & & & & & & & & & & $\uparrow 33 \%$ & \\
\hline Alanine & & & & $\uparrow 24 \%$ & & & & & & & & & & & \\
\hline myo-Inositol & $\uparrow 41 \%$ & & & & & & & & & & & & & & \\
\hline Taurine & & & & $\uparrow 28 \%$ & & & & & & & & & & & \\
\hline
\end{tabular}

This table summarizes significant NAC-induced metabolite changes relative to nonNAC-treated controls within the same genotype. Arrows indicate the direction of the NAC-induced change (i.e., arrows pointing down indicate that NAC reduced the metabolite concentration in animals of the same genotype and vice versa). One arrow represents $.005<p<.05$. Two arrows reflect $p<.005$. Note that most NAC-induced changes occur at early postnatal ages P10 and P20. P30 is characterized by a relative lack of NAC effects.

Gln, glutamine; Glu, glutamate; GPC/PCho, glycerophosphocholine/phosphocholine; $\mathrm{HZ}$, heterozygous; KO, knockout; NAC, N-acetylcysteine; $\mathrm{P}$, postnatal age; WT, wild type.

over time at multiple neurochemical levels that account for some but not all deficits, as evidenced by late Lac and Ala accumulation.

\section{Effects of NAC Treatment on GSH Levels}

Chronic NAC treatment increased GSH levels in the blood of WT offspring at P10 when pups were drinking the dam's milk, but not at P30. This indicates for the first time that NAC effects can be transmitted via the dam's milk. The fact that NAC increased blood GSH levels at P10 but not P30 may indicate that the early postnatal period is characterized by a relative lack of cysteine (Cys) availability compared with later ages. This is in accordance with the rise of GSH levels from P10 to 30 in nontreated animals of all genotypes. In addition, this age-dependent NAC effect could be related to a maturational change in the GSH inhibitory feedback control (35). The blood (and brain) of GCLM-KO mice showed no NAC-induced increase in GSH levels. This was expected because the GSH synthesizing enzyme GCL is hypofunctional in these animals (12).

In contrast to blood, there was no detectable NAC-induced increase of GSH in WT cortex. It has been demonstrated that NAC or NAC-derived Cys crosses the blood-brain barrier (76) to boost brain GSH levels during periods of increased oxidative stress $(76,77)$. Here we quantified cortical GSH levels upon NAC treatment at resting state rather than under increased stress condition. Because brain GSH levels are tightly regulated by a feedback inhibition system (35), NAC-induced rise in GSH levels were likely subtle and thus difficult to capture in cortex.

\section{NAC Treatment Normalizes Early Neurochemical Alterations in GCLM-HZ and KO Mice}

NAC crosses the placental barrier (78) and is transmitted via the dam's milk, suggesting that offspring of treated females benefited from NAC effects throughout the gestational and early postnatal period. Accordingly, NAC treatment normalized most neurochemical alterations in $\mathrm{KO}$ and $\mathrm{HZ}$ animals at P20. Clear examples of normalized metabolites are Gln, Glu, Gln/Glu, NAA, and Ins. KO animals also profited from NAC treatment at adult age, as indicated by the normalization of Ala at P90. Clinical studies show that NAC as adjunct to antipsychotic medication increases blood GSH levels and improves negative symptoms and medication side effects $(37,38)$. This is the first report that chronic NAC treatment normalizes cortical neurochemical abnormalities in a preclinical schizophrenia model.

How can NAC exert normalizing effects in GCLM-KO mice when NAC-derived Cys cannot be incorporated into GSH? A recent study demonstrates that NAC-derived Cys can enter an alternative redox system through which it exerts direct antioxidant and redoxregulatory actions (36). Specifically, Cys together with its oxidized form cystine (Cys) ${ }_{2}$ enters the (Cys) ${ }_{2} /$ Cys redox cycle, which is driven by the (Cys) ${ }_{2}$ /Glu antiporter (system $x_{c}^{-}$) (36). Interestingly, system $\mathrm{x}_{\mathrm{c}}{ }^{-}$has also been proposed to be an important mediator for the efficacy of NAC in improving negative symptoms in patients (37). Specifically, it has been proposed that NAC-derived Cys activates system $\mathrm{x}_{\mathrm{c}}{ }^{-}$, resulting in high extracellular Glu, which then activates extrasynaptic mGluR-2 receptors and leads eventually to less Glu release from pyramidal neurons (55). Thus, in GCLM-KO mice, NAC normalization may be mediated in part by system $\mathrm{x}_{\mathrm{c}}{ }^{-}$either through activation of mGluRs or through the (Cys) ${ }_{2} /$ Cys redox cycle, which can compensate part of the GSH deficit.

\section{Treatment with NAC Induces Changes in the Neurochemical Profile of All GCLM Genotypes}

Within-genotype analyses revealed that NAC treatment modified the cortical neurochemical profile of all genotypes, including

Table 3. GSH Levels in Whole Blood

\begin{tabular}{|c|c|c|c|c|}
\hline & \multicolumn{2}{|c|}{ P10 } & \multicolumn{2}{|c|}{ P30 } \\
\hline & Nontreated $\mu \mathrm{mol} / \mathrm{mL}$ & NAC-Treated $\mu \mathrm{mol} / \mathrm{mL}$ & Nontreated $\mu \mathrm{mol} / \mathrm{mL}$ & NAC-Treated $\mu \mathrm{mol} / \mathrm{mL}$ \\
\hline WT & $.37 \pm .06$ & $.73 \pm .03^{b b}$ & $.74 \pm .07^{c c}$ & $.62 \pm .08$ \\
\hline $\mathrm{HZ}$ & $.37 \pm .07$ & $.49 \pm .08$ & $.78 \pm .05^{c c}$ & $.69 \pm .05$ \\
\hline KO & $.12 \pm .02^{a a}$ & $.14 \pm .07^{a a}$ & $.38 \pm .08^{a a, c}$ & $.28 \pm .07^{a a}$ \\
\hline
\end{tabular}

Mean GSH concentrations \pm SEM are shown for each group. One superscript letter represents $.005<p<.05$. Two identical letters reflect $p<.005$.

$\mathrm{GSH}$, glutathione; $\mathrm{HZ}$, heterozygous; KO, knockout; NAC, N-acetylcysteine; P, postnatal age; WT, wild type.

${ }^{a}$ Significant difference of GSH concentrations with respect to WT.

${ }^{b}$ Significant increase in GSH concentration under NAC treatment.

'Significant difference in GSH concentration of nonNAC-treated animals between P10 and P30. 
WT. This emphasizes the importance of regular monitoring of NAC treatment effects in human subjects, particularly in young at-risk individuals who are not yet diagnosed with schizophrenia. NAC effects in WT occurred especially at early ages P10 and P20. Increased concentrations of Ala and Tau and the pronounced decrease of Gln and GIn/Glu suggest stimulation of glycolysis under NAC treatment (68). The pronounced increase in Tau during early development reflects conversion of NAC-derived cysteine to Tau (79).

\section{Conclusion}

Our data corroborate the role of oxidative stress and redox dysregulation in the development of schizophrenia and add to the validity of the GCLM-KO mouse model. Moreover, our data suggest that the prepubertal period is particularly sensitive to redox-related neurochemical changes, highlighting the importance of early monitoring and intervention. We suggest that an elevation of Gln, Glu, or GIn/Glu as observed in brains of early schizophrenia patients may already be present at prepubertal age and may thus qualify as potential early biomarker for schizophrenia in the future. NAC may be a promising candidate for early treatment intervention. Finally, our data highlight the translational value of MRS. This technique has been implemented on clinical platforms to allow the quantification of neurochemical profiles comprising 14 metabolites. Here we show that the same technique can be reliably applied in a preclinical schizophrenia model to quantify the same 14 (plus 4) metabolites. This opens up new avenues for future translational research.

This work was supported by the Centre d'Imagerie BioMédicale of the University of Lausanne, University of Geneva, University Hospital Geneva, University Hospital Lausanne, Ecole Polytechnique Federale Lausanne, and the Leenaards and Jeantet Foundations; the Swiss National Science Foundation (Grant No. 310030-135736 to KQD) and Loterie Romande.

We thank the National Center of Competence in Research (NCCR) "SYNAPSY - The Synaptic Bases of Mental Diseases." We are also grateful to Adeline Cottier and Hélène Moser for technical assistance.

The authors report no biomedical financial interests or potential conflicts of interest.

1. Cookson MR, Bandmann O (2010): Parkinson's disease: Insights from pathways. Hum Mol Genet 19(R1): R21-27.

2. Agostinho P, Cunha RA, Oliveira C (2010): Neuroinflammation, oxidative stress and the pathogenesis of Alzheimer's disease. Curr Pharm Des $16: 2766-2778$

3. Turner C, Schapira AH (2010): Mitochondrial matters of the brain: The role in Huntington's disease. J Bioenerg Biomembr 42:193-198.

4. Steckert AV, Valvassori SS, Moretti M, Dal-Pizzol F, Quevedo J (2010): Role of oxidative stress in the pathophysiology of bipolar disorder. Neurochem Res 35:1295-1301.

5. Do KQ, Cabungcal J-H, Frank A, Steullet P, Cuenod M (2009): Redox dysregulation, neurodevelopment, and schizophrenia. Curr Opin Neurobiol 19:220-230.

6. Do KQ, Trabesinger AH, Kirsten-Krüger M, Lauer CJ, Dydak U, Hell D, et al. (2000): Schizophrenia: Glutathione deficit in cerebrospinal fluid and prefrontal cortex in vivo. Eur J Neurosci 12:3721-3728.

7. Matsuzawa D, Hashimoto K (2011): Magnetic resonance spectroscopy studies of the antioxidant system in schizophrenia. Antioxid Redox Signal 15:2057-2065.

8. Yao JK, Leonard S, Reddy R (2006): Altered glutathione redox state in schizophrenia. Dis Markers 22:83-93.

9. Tosic M, Ott J, Barral S, Bovet P, Deppen P, Gheorgita F, et al. (2006): Schizophrenia and oxidative stress: Glutamate cysteine ligase modifier as a susceptibility gene. Am J Hum Genet 79:586-592.
10. Gysin R, Kraftsik R, Sandell J, Bovet P, Chappuis C, Conus P, et al. (2007): Impaired glutathione synthesis in schizophrenia: Convergent genetic and functional evidence. Proc Natl Acad Sci U S A 104:16621-16626.

11. Yao JK, Reddy R (2011): Oxidative stress in schizophrenia: Pathogenetic and therapeutic implications. Antioxid Redox Signal 15:1999-2002.

12. Yang Y, Dieter MZ, Chen Y, Shertzer HG, Nebert DW, Dalton TP (2002): Initial characterization of the glutamate-cysteine ligase modifier subunit Gclm(-/-) knockout mouse: Novel model system for a severely compromised oxidative stress response. J Biol Chem 277:49446-49452.

13. Steullet $P$, Cabungcal J-H, Kulak A, Kraftsik R, Chen $Y$, Dalton TP, et al (2010): Redox dysregulation affects the ventral but not dorsal hippocampus: Impairment of parvalbumin neurons, gamma oscillation, and related behaviours. J Neurosci 30:2547-2558.

14. Do KQ, Steullet P, Conus P, Kulak A, Cuenod M, Cabungcal J-H (2010): Reverse translational approach to redox dysregulation in schizophrenia: Genetics, environment, neurodevelopment and therapy. Biol Psychiatry 67(suppl):199.

15. Monin A, Klaey M, Butticaz C, Cabungcal JH, Steullet P, Do KQ (2011): Redox dysregulation affects proliferation, differentiation of oligodendrocyte progenitors and myelination: Relevance for Schizophrenia Abstract at the Viewer/Itinerary Planner, 41th Annual Meeting of the Society for Neuroscience, Washington, DC.

16. Lewis DA, Levitt $P$ (2002): Schizophrenia as a disorder of neurodevelopment. Annu Rev Neurosci 25:409-432.

17. Rapoport JL, Gogtay N (2010): Childhood onset schizophrenia: Support for a progressive neurodevelopmental disorder. Int J Dev Neurosci 29: 251-258.

18. Steen RG, Hamer RM, Lieberman JA (2005): Measurements of brain metabolites by $1 \mathrm{H}$ magnetic resonance spectroscopy in patients with schizophrenia: A systematic review and methanalysis. Neuropsychology 30:1949-1962.

19. Klär AA, Ballmaier M, Leopold K, Häke I, Schaefer M, Brühl R, et al. (2010): Interaction of hippocampal volume and $\mathrm{N}$-acetylaspartate concentration deficits in schizophrenia: A combined MRI and 1H-MRS study. Neuroimage 53:51-57.

20. Goto N, Yoshimura R, Kakeda S, Moriya J, Hayashi K, Ikenouchi-Sugita A, et al. (2011): Comparison of brain $N$-acetylaspartate levels and serum brain-derived neurotrophic factor (BDNF) levels between patients with first-episode schizophrenia psychosis and healthy controls. Eur Psychiatry 26:57-63.

21. Keshavan MS, Dick RM, Diwadkar VA, Montrose DM, Prasad KM, Stanley JA (2009): Striatal metabolic alterations in non-psychotic adolescent offspring at risk for schizophrenia: A (1)H spectroscopy study. Schizophr Res 115:88-93.

22. Yoo SY, Yeon S, Choi C-H, Kang D-H, Lee J-M, Shin NY, et al. (2009): Proton magnetic resonance spectroscopy in subjects with high genetic risk of schizophrenia: Investigation of anterior cingulated, dorsolateral prefrontal cortex and thalamus. Schizophr Res 111:86-93.

23. Bustillo JR, Rowland LM, Mullins P, Jung R, Chen H, Qualls C, et al. (2009): ${ }^{1} \mathrm{H}-\mathrm{MRS}$ at 4 Tesla in minimally treated early schizophrenia. Mol Psychiatry 15:629-636.

24. Lutkenhoff ES, van Erp TG, Thomas MA, Therman S, Manninen M, Huttunen MO, et al. (2008): Proton MRS in twin pairs discordant for schizophrenia. Mol Psychiatry 15:308-318.

25. Tibbo P, Hanstock C, Valiakalayil A, Allen P (2004): 3-T proton MRS investigation of glutamate and glutamine in adolescents at high genetic risk for schizophrenia. Am J Psychiatry 161:1116-1118.

26. Hashimoto K, Engberg G, Shimizu E, NordinC, Lindström LH, lyo M (2005): Elevated glutamine/glutamate ratio in cerebrospinal fluid of first episode and drug naïve schizophrenic patients. BMC Psychiatry 5:6.

27. de la Fuente-Sandoval C, León-Ortiz P, Favila R, Stephano S, Mamo D, Ramírez-Bermúdez J, et al. (2011): Higher levels of glutamate in the associative-striatum of subjects with prodromal symptoms of schizophrenia and patients with first-episode psychosis. Neuropsychopharmacology 36:1781-1791.

28. Tayoshi SY, Sumitani S, Taniguchi K, Shibuya-Tayoshi S, Numata S, Iga J, et al. (2009): Metabolite changes and gender differences in schizophrenia using 3-Tesla proton magnetic resonance spectroscopy ( ${ }^{1} \mathrm{H}-\mathrm{MRS}$ ). Schizophr Res 108:69-77.

29. Ohrmann P, Siegmund A, Suslow T, Spitzbergs K, Kersting K, Rothermundt $M$, et al. (2005) Evidence for glutamatergic neuronal dysfunction in the prefrontal cortex in chronic but not first-episode patients with 
schizophrenia: A proton magnetic resonance spectroscopy study Schizophr Res 73:153-157.

30. Iltis I, Koski DM, Eberly LE, Nelson CD, Deelchand DK, Valette J, et al. (2009): Neurochemical changes in the rat prefrontal cortex following acute phencyclidine treatment: An in vivo localized ${ }^{1} \mathrm{H}$ MRS study. NMR Biomed 22:737-744.

31. Krystal JH, Karper LP, Seibyl JP, Freeman GK, Delaney ER, Bremner JD, et al. (1994): Subanesthetic effects of the noncompetitive NMDA antagonist, ketamine, in humans, Psychomimetic, perceptual, cognitive, and neuroendocrine responses. Arch Gen Psychiatry 51:199-214.

32. Kantrowitz JT, Javitt DC (2010): Thinking glutamatergically: Changing concepts of schizophrenia based upon changing neurochemical models. Clin Schizophr Relat Psychoses 4:189-200.

33. Kulak A, Duarte JMN, Do KQ, Grütter R (2010): Neurochemical profile of the developing mouse cortex determined by in vivo ${ }^{1} \mathrm{H}$ NMR spectroscopy at $14 \mathrm{~T}$ and the effect of recurrent anesthaesia. J Neurochem 115: $1466-1477$.

34. Atkuri KR, Mantovani JJ, Herzenberg LA, Herzenberg LA (2007): N-Acetylcysteine-A safe antidote for cysteine/glutathione deficiency. Curr Opin Pharmacol 7:355-359.

35. Meister A (1995): Glutathione biosynthesis and its inhibition. Methods Enzymol 252:26-30.

36. Mandal PK, Seiler A, Perisic T, Kölle P, Banjac Canak A, Förster H, et al. (2010): System x(c)- and thioredoxin reductase 1 cooperatively rescue glutathione deficiency. J Biol Chem 285(29): 22244-53.

37. Berk M, Copolov D, Dean O, Lu K, Jeavons S, Schapkaitz I, et al. (2008): $\mathrm{N}$-acetyl cysteine as a glutathione precursor for schizophrenia-a double-blind, randomized, placebo-controlled trial. Biol Psychiatry 64:361368.

38. Lavoie $S$, Murray MM, Deppen $P$, Knyazeva MG, Berk M, Bovet $P$, et al. (2008): Glutathione precursor, $N$-acetyl-cysteine, improves mismatch negativity in schizophrenia patients. Neuropsychopharmacology 33 2187-2199.

39. Hedrich H, Bullock G, Petrusz P (2008): The Handbook of Experimental Animals: The Laboratory Mouse. Oxford, England: Academic Press.

40. Mekle R, Mlynárik V, Gambarota G, Hergt M, Krueger G, Gruetter R (2009): MR spectroscopy of the human brain with enhanced signal intensity at ultrashort echo times on a clinical platform at 3T and 7T. Magn Reson Med 61:1279-1285.

41. Gambarota G, Mekle R, Xin L, Hergt M, van der Zwaag W, Krueger G, et al. (2008): In vivo measurement of glycine with short echo-time $1 \mathrm{H}$ MRS in human brain at 7 T. MAGMA 22:1-4.

42. Pfeuffer J, Tkác I, Provencher SW, Gruetter R (1999): Toward an in vivo neurochemical profile: Quantification of 18 metabolites in short-echotime (1)H NMR spectra of the rat brain. J Magn Reson141:104-120.

43. Gruetter R (1993): Automatic, localized in vivo adjustment of all firstand second-order shim coils. Magn Reson Med 29:804-811.

44. Gruetter R, Tkáč I (2000): Field mapping without reference scan using asymmetric echo-planar techniques. Magn Reson Med 43:319-323.

45. Mlynárik V, Gambarota G, Frenkel H, Gruetter R (2006): Localized shortecho-time proton MR spectroscopy with full signal-intensity acquisition. Magn Reson Med 56:965-970.

46. Bachmanov AA, Reed DR, Beauchamp GK, Tordoff MG (2002): Food intake, water intake, and drinking spout side preference of 28 mouse strains. Behav Genet 32:435-443.

47. Montgomery DC, Peck EA, Vining GG (2006): Introduction to Linear Regression Analysis, 4th edition. Chichester, England.

48. Koga M, Messmer M, Sawa A, Snyderr SH, Sedlak TW (2010): Glutathione un-GLU-ed: A role for glutathione in neuronal glutamate metabolism. Abstract 225.6 Viewer/Itinerary Planner, Society for Neuroscience, San Diego, California.

49. Miao Y, Qiu Y, Lin Y, Miao Z, Zhang J, Lu X (2010): Protection by pyruvate against glutamate neurotoxicity is mediated by astrocytes through glutathione-dependent mechanism. Mol Biol Rep 38:3235-3242.

50. Hertz J (2006): Glutamate, a neurotransmitter-and so much more. A synopsis of Wierzba III. Neurochem Int 48:416-425.

51. Kantrowitz JT, Javitt DC (2010): N-methyl-d-aspartate (NMDA) receptor dysfunction or dysregulation: The final common pathway on the road to schizophrenia? Brain Res Bull 83:108-121.

52. Homayoun H, Moghaddam B (2007): NMDA receptor hypofunction produces opposite effects on prefrontal cortex interneurons and pyramidal neurons. J Neurosci 27:11496-11500.
53. Cuenod M, Cabungcal J-H, Kulak A, Steullet P, Do KQ (2011): Redox dysregulation and oxidative stress affect parvalbumin interneurons and neural synchrony in anterior cingulated cortex during neurodevelopment. Schizophr Bull 37(suppl 1):102.

54. Patil ST, Zhang L, Martenyi F, Lowe SL, Jackson KA, Andreev BV, et al. (2007): Activation of $\mathrm{mGlu2} / 3$ receptors as a new approach to treat schizophrenia: A randomized Phase 2 clinical trial. Nat Med 13:11021107.

55. Krystal JH (2008): Capitalizing on extrasynaptic glutamate neurotransmission to treat antipsychotic-resistant symptoms in schizophrenia [review]. Biol Psychiatry 64:358-360.

56. Torres GE, Pena K, Quiroz M, Amara SG, Underhill SM (2010): Functional coupling between the glutamate transporter EAAT2 and glutamine synthetase. Abstract 544.13 Viewer/ltinerary Planner, 40th Annual Meeting of the Society for Neuroscience, San Diego, California.

57. Costantino-Ceccarini E, Morell P (1972): Biosynthesis of brain sphingolipids and myelin accumulation in the mouse. Lipids 7:656-659.

58. Muse ED, Jurevics H, Toews AD, Matsushima GK, Morell P (2001): Parameters related to lipid metabolism as markers of myelination in mouse brain. J Neurochem 76:77-86.

59. Kirmani BF, Jacobowitz DM, Kallarakal AT, Namboodiri MA (2002): Aspartoacylase is restricted primarily to myelin synthesizing cells in the CNS: Therapeutic implications for Canavan disease. Brain Res Mol Brain Res 107:176-182.

60. Höistad M, Segal D, Takahashi N, Sakurai T, Buxbaum JG, Hof PR (2009): Linking white and grey matter in schizophrenia: Oligodendrocyte and neuron pathology in the prefrontal cortex. Front Neuroanat 3:9.

61. Smith J, Ladi E, Mayer-Proschel M, Noble M (2000): Redox state is a central modulator of the balance between self-renewal and differentiation in a dividing glial precursor cell. Proc Natl Acad Sci U S A 97:1003210037.

62. Yao J, Stanley JA, Reddy RD, Keshavan MS, Pettegrew JW (2002): Correlations between peripheral polyunsaturated fatty acid content and in vivo membrane phospholipid metabolites. Biol Psychiatry 52:823-830.

63. Pettegrew JW, Keshavan MS, Panachalingam K, Strychor S, Kaplan DB, et al. (1991): Alterations in brain high-energy phosphate and membrane phospholipid metabolism in first-episode, drug-naïve schizophrenics: A pilot study of the dorsal prefrontal cortex by in vivo phosphorus 31 nuclear magnetic resonance spectroscopy. Arch Gen Psychiatry 48:563568.

64. Bustillo JR, Chen H, Gasparovic C, Mullins P, Caprihan A, Qualls C, et al. (2011): Glutamate as a marker of cognitive function in schizophrenia: $A$ proton spectroscopic imaging study at 4 Tesla. Biol Psychiatry 69:19-27.

65. Chang L, Ernst T, Witt MD, Ames N, Gaiefsky M, Miller E (2002): Relationships among brain metabolites, cognitive function, and viral loads in antiretroviral-naïve HIV patients. Neuroimage 17:1638-1648.

66. Chantal S, Labelle M, Bouchard RW, Braun CM, Boulanger Y (2002): Correlation of regional proton magnetic resonance spectroscopic metabolic changes with cognitive deficits in mild Alzheimer disease. Arch Neurol 59:955-962.

67. Parnetti L, Tarducci R, Presciutti O, Lowenthal DT, Pippi M, Palumbo B, et al. (1997): Proton magnetic resonance spectroscopy can differentiate Alzheimer's disease from normal aging. Mech Ageing Dev 97:9-14.

68. McLain AL, Szweda PA, Szweda LI (2011): $\alpha$-ketoglutarate dehydrogenase: A mitochondrial redox sensor. Free Radic Res 45:29-36.

69. Halim ND, Lipska BK, Hyde TM, Deep-Soboslay A, Saylor EM, Herman MM, et al. (2008): Increased lactate levels and reduced pH in postmortem brains of schizophrenics: Medication confounds. J Neurosci Methods 169:208-213.

70. Beasley CL, Dwork AJ, Rosoklija G, Mann J, Mancevski B, Jakovski Z, et al. (2009): Metabolic abnormalities in fronto-striatal-thalamic white matter tracts in schizophrenia. Schizo Res 109:159-166.

71. Prabakaran S, Swatton JE, Ryan MM, Huffaker SJ, Huang JT, Griffin JL, et al. (2004): Mitochondrial dysfunction in schizophrenia: Evidence for compromised brain metabolism and oxidative stress. Mol Psychiatry 9:684-97, 643.

72. Regenold WT, Phatak P, Marano CM, Sassan A, Conley RR, Kling MA (2009): Elevated cerebrospinal fluid lactate concentrations in patients with bipolar disorder and schizophrenia: Implications for the mitochondrial dysfunction hypothesis. Biol Psychiatry 65:489-494.

73. Holmes E, Tsang TM, Huang JT, Leweke FM, Koethe D, Gerth CW, et al. (2006): Metabolic profiling of CSF: Evidence that early intervention may impact on disease progression and outcome in schizophrenia. PLoS Med 3:e327. 
74. Hatano T, Ohnuma T, Sakai Y, Shibata N, Maeshima H, Hanzawa R, et al (2010): Plasma alanine levels increase in patients with schizophrenia as their clinical symptoms improve-Results from the Juntendo University Schizophrenia Projects (JUSP). Psychiatry Res 177:27-31.

75. De Graaf-Peters VB, Hadders-Algra M (2006): Ontogeny of the human nervous system: What is happening when? Early Hum Dev 82:257-266.

76. Farr SA, Poon HF, Dogrukol-Ak D, Drake J, Banks WA, Eyerman E, et al. (2003): The antioxidants alpha-lipoic acid and $\mathrm{N}$-acetylcysteine reverse memory impairment and brain oxidative stress in aged SAMP8 mice. J Neurochem 84:1173-1183.
77. Lanté F, Meunier J, Guiramand J, De Jesus Ferreira MC, Cambonie G Aimar R, et al. (2008): Late $\mathrm{N}$-acetylcysteine treatment prevents the deficits induced in the offspring of dams exposed to an immune stress during gestation. Hippocampus 18:602-609.

78. Izzotti A, Balansky RM, Camoirano A, Cartiglia C, Longobardi M, Tampa E, et al. (2003): Birth-related genomic and transcriptional changes in mouse lung modulation by transplacental $\mathrm{N}$-acetylcysteine. Mutat Res 544:441-449.

79. Schuller-Levis GB, Park E (2003): Taurine: New implications for an old amino acid. FEMS Microbiol Lett 226:195-202. 